\title{
„Einen 100. Kongress gibt es wirklich nur einmal - da sollte man dabei sein!“
}

\section{Interview mit Professor Michael Forsting, Kongresspräsident des 100. Röntgenkongresses der Deutschen Röntgengesellschaft in Leipzig}

\begin{abstract}
Vom 29. Mai bis 1. Juni 2019 findet in Leipzig zum 100. Mal der Röntgenkongress der Deutschen Röntgengesellschaft (DRG) statt. Einer der drei Kongresspräsidenten ist Professor Dr. med. Michael Forsting, Direktor der Universitätsradiologie Essen. Im Interview spricht er über das Kongressthema „Radiologie 4.0“ und die Bedeutung der Digitalisierung für das Fach. In seinem Essay spannt Professor Forsting den Bogen von der Künstlichen Intelligenz in der Radiologie visionär weiter - hin zu Zentren für Super-Diagnostics: eine Ermutigung, künftige Technologien aktiv mitzugestalten.
\end{abstract}

Das Motto des 100. Röntgenkongresses lautet „Einheit in Vielfalt“ - worauf zielt es $a b$ ?

„Einheit in Vielfalt“ soll zeigen, wie vielseitig die Radiologie ist. Wir drei Präsidenten stehen für drei unterschiedliche Schwerpunkte in der Radiologie: Herr Professor Heindel ist Allgemeinradiologe, Frau Professor Staatz ist Kinderradiologin und ich bin Neuroradiologe. Wir gehören aber trotzdem alle einem Fach an. In den vergangenen 40 Jahren wurde häufiger diskutiert, dass man die Radiologie komplett zerschlagen und eine reine Organradiologie aufbauen sollte. Diese Diskussion hat sich wieder beruhigt, sodass wir das Motto „Einheit in Vielfalt“ gewählt haben.

Welchen der fachlichen Schwerpunkte haben Sie als Kongresspräsident geprägt?

Ich bin Neuroradiologe, habe aber nicht das Gehirn zum Schwerpunkt gemacht, sondern mit dem Thema „Radiologie 4.0“ auf die Digitalisierung der Radiologie fokussiert. Mit Digitalisierung meine ich die Möglichkeit, mit den uns vorliegenden Daten bessere Diagnosen zu stellen, wenn wir beispielsweise künstliche Intelligenz (KI) zur Hilfe nehmen.

\section{Warum sollten Ihre Kollegen sich auf den Weg nach Leipzig machen?}

Einen 100. Kongress gibt es wirklich nur einmal, und da sollte man dabei sein. Wenn die Röntgenstrahlen schon in Deutschland entdeckt worden sind und wir es als erste nationale Gesellschaft für Radiologie schaffen, einen 100. Kongress auf die Beine zu stellen - also ich würde da nicht fehlen wollen!

\section{Worauf freuen Sie sich besonders bei diesem Kongress?}

Ich freue mich auf die Highlights, die wir Präsidenten in den einzelnen Schwerpunkten und Eröffnungsvorträgen gesetzt haben. Ich hoffe, dass viele Chefs ihre jungen Kolleginnen und Kollegen für die eine Woche freistellen, damit sie die Gelegenheit haben, nach Leipzig zu kommen - denn das Weiterbildungsprogramm ist schon ziemlich gut. Und dann gibt es bei jedem Kongress ja auch gesellschaftliche Ereignisse. Beim 100. Kongress haben wir uns gedacht, wir feiern jetzt einmal mit 7000 Leuten - das hat es so noch nicht gegeben.

In Ihrem Schwerpunkt „Radiologie 4.0“ wird es um das Thema KI, Künstliche Intelligenz, gehen. Was verstehen Sie darunter?

Das Wort „Künstliche Intelligenz“ ist in den zurückliegenden zwei bis drei Jahren ein häufig verwendetes Schlagwort geworden. Es steht letztlich für eine ziemlich heterogene Gruppe von Algorithmen, die in der Lage sind zu lernen. In der Radiologie setzen wir sehr viel Software ein. Bislang wird diese Software programmiert beziehungsweise modelliert: Wir müssen mathematische Formeln finden, um bestimmte Dinge zu beschreiben. Die KI-Algorithmen hingegen werden am Ergebnis trainiert. Wir zeigen einem KI-Algorithmus zum Beispiel eine Mammografie und sagen ihm: Das ist ein Mammakarzinom, ein Brustkrebs. Den Vorgang wiederholen

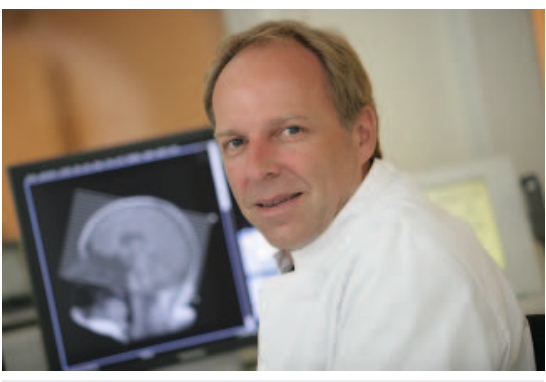

Prof. Dr. med. Michael Forsting

wir mit etwa 1000 Bildern. Dann hat dieser Algorithmus eigenständig gelernt, wie ein solches Mammakarzinom aussieht. Für viele mag das eine seltsame Vorstellung sein, funktioniert aber extrem gut. Der Erfolg von $\mathrm{KI}$ in desr Radiologie hängt davon $a b$, wie valide die Daten sind, die vorliegen, um Algorithmen zu trainieren. Im Moment haben wir die Situation, dass die großen Internetplayer wie Google, Amazon oder Apple zwar über Algorithmen verfügen, nicht aber über radiologische Daten. Deshalb wollen wir im Lauf des Kongresses auch herausfinden, was wir Radiologen beim Thema KI selbst entwickeln und auf den Weg bringen können.

Welche Rolle spielt der Datenschutz dabei?

Datenschutz ist insgesamt ein wichtiges Thema in Deutschland und Europa. Aber es gilt: Wir haben bei KI kein Datenschutzproblem, solange wir unsere radiologischen Daten nicht an Apple, Amazon oder Google verkaufen. Wenn eine radiologische Abteilung selbst Algorithmen trainiert, diese dann intelligent werden und ein Mammakarzinom erkennen können, sind am Ende gar keine Patientendaten mehr enthalten. Das Problem Datenschutz entfällt somit.

Was kommt auf die Radiologen mit dem Einsatz von KI zu? Müssen sie sich sehr umstellen?

Die Angst der Radiologen vor einer Abschaffung der Radiologie ist völlig irratio- 
nal. Das Gegenteil ist der Fall: Wir werden noch besser. Wenn man ganz allgemein sagen würde, wir geben dem Radiologen Werkzeuge an die Hand, damit er besser und schneller wird, wäre Angst gar kein Thema. Nur weil man jetzt über Künstliche Intelligenz diskutiert, die ihn besser und schneller macht, fangen einige an zu zittern. Das ergibt natürlich keinen Sinn. Man kann KI in der Radiologie mit dem Autopiloten im Airbus vergleichen. Natürlich waren Piloten vor seiner Erfindung in Notsituationen stärker gefordert. Trotzdem kann man sich ja nicht vorstellen, dass heute ein Flugzeug ohne Piloten aus Fleisch und Blut fliegt - auf den Plausibilitätscheck durch Menschenverstand möchte man nicht verzichten. So ähnlich wird es sich auch in der Radiologie verhalten. Man wird nicht alle Krankheiten mit KI diagnostizieren können, aber man wird mit $\mathrm{KI}$ in der Diagnostik besser werden und Krankheiten besser verstehen. Ich glaube, dass die sprechende Medizin durch KI ganz anders herausgefordert wird, weil in der sprechenden Medizin mehr Fehler möglich sind. Wenn ein radiologisches Bild entsteht und einen Tumor in der Leber mit 3,5 Zentimetern Größe zeigt, dann wird es sich schon um einen Tumor von 3,5 Zentimetern Größe handeln. Die Tatsache, dass da ein Tumor ist, wird man nicht infrage stellen. Wenn jemand hingegen mit Rückenschmerzen zum Allgemeinarzt geht, ist es eher zweifelhaft, ob der Allgemeinarzt am Ende eine Depression diagnostiziert. Das wird mit KI künftig sehr viel sicherer gehen. Es gibt bereits Algorithmen, die anhand einer Stimme analysieren können, ob jemand depressiv ist. Das ist auch gar nicht verwunderlich, weil ein guter Psychiater das auch macht. Also: Uns Radiologen wird es weiterhin geben.

\section{Welche Anwendungen von intelligenter Software gibt es bereits im klinischen Alltag?}

Es gibt ja in der Radiologie durchaus auch langweilige Tätigkeiten, zum Beispiel das Bestimmen des Knochenalters bei kindlichen Entwicklungsstörungen. Daran hat niemand wirklich Freude. Es gibt bereits Lizenzmodelle, mit deren Hilfe ein Computer das analysiert. Natürlich schaut man noch einmal darauf, um nicht etwa zu übersehen, dass die Hand gebrochen ist.
Das kann dieser Algorithmus nämlich nicht erkennen. Aber man muss nicht mehr dicke Bücher wälzen, um herauszufinden, wie alt der Knochen tatsächlich ist. Ähnliche Algorithmen gibt es auch schon für Schlaganfalldiagnostik und für die Mammografie. Das sind kommerzielle Produkte, für die die amerikanische Regulierungsbehörde Food and Drug Administration (FDA) ein Standardverfahren (Standard Operating Procedure, kurz: SOP) entwickelt hat, mit dem man solche Softwarelösungen zulassen kann.

\section{Wir haben jetzt viele Vorteile von KI-An- wendungen gehört. Gibt es auch Nach- teile?}

Alles hat Vor- und Nachteile. Man könnte auch über die Nachteile der Magnetresonanztomografie nachdenken: Die Untersuchungszeiten sind etwas länger, man muss in einem Tunnel liegen ... Das stimmt alles, aber gemessen an den Vorteilen ist das kein Nachteil. Wenn man über Softwarelösungen mit KI nachdenkt, könnte einem einfallen: Der Radiologe lernt gar nicht mehr, das Knochenalter zu bestimmen, weil das immer die Software macht. Das stimmt, aber als ich studiert habe, musste man auch noch den Herzfehler mit einem Stethoskop erkennen. Das muss heute keiner mehr, weil man inzwischen weiß, dass dabei eine Menge falsch war. Ist es jetzt ein Nachteil, dass ein Arzt heute kein Stethoskop mehr benutzt? Ich glaube nicht. Aber es wird sicher sehr schlaue Menschen geben, die einen Nachteil finden.

\section{Kann es Fehldiagnosen geben?}

Es wird immer Fehldiagnosen geben. Denn die Annahme, dass ein Bild immer nur ganz spezifisch für eine Krankheit stehen muss, ist falsch. Manchmal weiß man nicht genau, ob das Bild wirklich nur eine Erkrankung oder nicht vielleicht doch zwei oder drei Erkrankungen zeigt. Das ist wie mit Husten. Sie können husten, weil Sie sich gerade verschluckt haben, oder weil Sie unter einer Viruserkrankung oder einem Lungentumor leiden. Insofern können Sie auch mit dem Symptom Husten nicht immer auf nur eine Erkrankung schließen. Genauso verhält es sich mit dem Bild. Algorithmen sind immer nur so gut wie die Daten, mit denen sie trainiert werden.
Und wenn in den Daten ein Fehler steckt, dann hat dieser Algorithmus automatisch auch einen Fehler. Am Ende wird es aber so sein, dass die Algorithmen weniger Fehler machen als die Menschen - und genau das ist der wesentliche Vorteil. Wenn ein selbstfahrendes Auto in Kalifornien ein Kaninchen überfahren hat, dann steht das heute auf der Titelseite. Würde man das bei jedem Unfall machen, der durch menschliches Versagen entstanden ist, wäre die tägliche Ausgabe der Tageszeitung mehrbändig. Man muss schon richtig skalieren und prüfen: Wie viele Fehler machen wir als Radiologen, und wie viele Fehler macht die Maschine?

Wird sich das Arzt-Patienten-Verhältnis verändern, wenn solche Werkzeuge eingesetzt werden?

Das Arzt-Patienten-Verhältnis hat sich in der Radiologie durch die Einführung der Computer- oder der Magnetresonanztomografie auch nicht verändert. Im Gegenteil, vielleicht hat der Patient sogar mehr Vertrauen zu uns gewonnen, weil er merkt, dass wir schneller und besser geworden sind. Aber natürlich diskutiert man darüber: Wird Medizin unmenschlicher, wenn Computer die Diagnose übernehmen? Man muss sich aber auch die Frage stellen: Was wollen wir denn eigentlich als Patienten? Doch möglichst schnell eine richtige Diagnose. Es hilft uns überhaupt nicht, wenn ein äußerst empathischer Arzt nach 48 Stunden Überlegen eine falsche Diagnose stellt. Dann kommt es natürlich auch darauf an, wie man dem Patienten erklärt, wie krank er ist oder dass er vielleicht auch gar nicht krank ist. Aber die Tatsache, dass wir schneller und besser in unserer Diagnose werden, wird das Arzt-Patienten-Verhältnis eigentlich nur verbessern können.

\section{Wohin wird sich die Radiologie entwi- ckeln?}

Die diagnostischen Fächer müssen sich genau überlegen, wohin sie sich entwickeln wollen. Dazu zählen Radiologie, Nuklearmedizin, Pathologie und genau genommen auch alle Laborfächer sowie die Genetik. In diesen Fächern werden schnell viele Daten digital vorliegen. Da müssen wir uns fragen: Wie wollen wir in Zukunft 
kooperieren? Ich sage nicht, dass man die Fächergrenzen aufbrechen muss. Aber ich glaube schon, dass es Plattformen geben sollte, die alle Befunde so integrieren, dass man am Ende sehr individualisiert zu einer Diagnose kommt. Das ist bei Tumoren heute fast schon üblich. Man macht Tumorboards, an denen nicht nur der Radiologe teilnimmt, sondern auch der
Genetiker, der Pathologe und so weiter, weil es auf die Gesamtheit der Befunde ankommt. Und wir Radiologen sind eine große Berufsgruppe, wir könnten uns auf den Fahrersitz setzen, um eine Antwort zu finden auf die Frage: Wie erhalten wir eine Integration aller Befunde? Auch darüber werden wir auf dem 100. Röntgenkongress sprechen.
Der 100. Deutsche Röntgenkongress findet vom 29. Mai bis 1. Juni 2019 im Congress Center Leipzig statt. Weitere Informationen unter https:// www.roentgenkongress.de Einen Kurzfilm mit dem Kongresspräsidenten Professor Dr. med. Michael Forsting finden Sie online unter https://youtu.be/FPQRFCRM5Jg

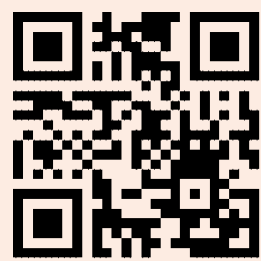

